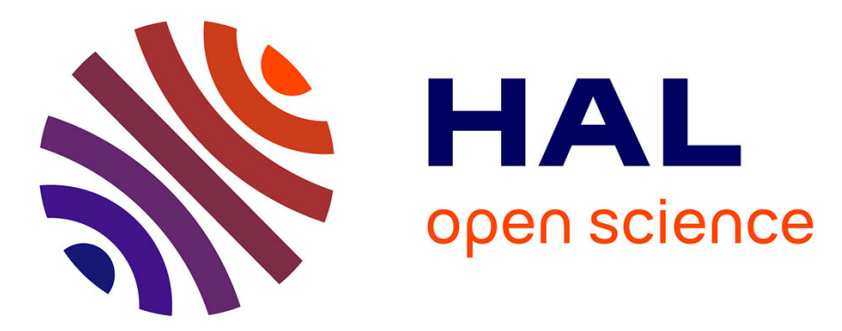

\title{
A Step Toward Mobile Robots Autonomy: Energy Estimation Models
}

Lotfi Jaïem, Sébastien Druon, Lionel Lapierre, Didier Crestani

\section{To cite this version:}

Lotfi Jaïem, Sébastien Druon, Lionel Lapierre, Didier Crestani. A Step Toward Mobile Robots Autonomy: Energy Estimation Models. TAROS 2016 - 17th Annual Conference Towards Autonomous Robotic Systems, Jun 2016, Sheffield, United Kingdom. pp.177-188, 10.1007/978-3-319-40379-3_18 . lirmm-01310961

\section{HAL Id: lirmm-01310961 https://hal-lirmm.ccsd.cnrs.fr/lirmm-01310961}

Submitted on 19 May 2016

HAL is a multi-disciplinary open access archive for the deposit and dissemination of scientific research documents, whether they are published or not. The documents may come from teaching and research institutions in France or abroad, or from public or private research centers.
L'archive ouverte pluridisciplinaire HAL, est destinée au dépôt et à la diffusion de documents scientifiques de niveau recherche, publiés ou non, émanant des établissements d'enseignement et de recherche français ou étrangers, des laboratoires publics ou privés. 


\title{
A Step Toward Mobile Robots Autonomy: Energy Estimation Models
}

\author{
Lotfi Jaiem, Sebastien Druon, Lionel Lapierre, and Didier Crestani
}

Authors are members of the Laboratoire d'Informatique Robotique et Microélectronique de Montpellier (LIRMM), UMR 5506, Université de Montpellier, 161 rue Ada, 34095 Montpellier Cedex 5, France.

\{jaiem, druon, lapierre, crestani\}@lirmm.fr

http://www.lirmm.fr

\begin{abstract}
One of the crucial questions to develop Autonomous Mobile Robotic systems is the energy consumption, its monitoring and management all along the mission. Mission complexity and fault tolerance capabilities require to exploit system redundancies, in terms of algorithms and hardware recruitment. This choice has an evident impact on energy consumption. This paper proposes an identification protocol to establish the energy consumption models for each control configuration of a Pioneer 3DX. These models are destined to be used as online predictors providing an estimation of the necessary remaining energy that mission success 'nominally' requires. At the end, the proposed energy consumption models are validated and discussed experimentally.
\end{abstract}

Keywords: Autonomy, energy, control architecture, consumption model

\section{Introduction}

Mobile robotic systems are expected to perform long range, long term and complex missions. By contrast to industrial robotics where energy can be supposed to be infinite, the management of autonomous mobile robot missions requires to be able predicting the energy consumption of the robot with an acceptable accuracy. Moreover, the mission complexity and the environment versatility imposes to be able managing the embedded energy according to different robot configurations (sensors, actuators, control schemes,etc.), making the energy management a central issue for autonomous robotic missions. Related research addressing the energy issue can be classified into 3 main fields.

Firstly, component oriented approaches like Dynamic Power Management (DPM) in [1], want to reduce the energy consumption using hardware or software energy-aware management techniques. An estimation of the future activity of hardware and software components is used to dynamically adjust their power, while maintaining a desired performance. One of the most popular DPM techniques is the Dynamic Voltage Scaling (DVS) which dynamically adapts the frequency and the voltage of the processor for energy saving [2]. These approaches 
can be extended to robotics, for power supply management, as example, scaling the sensors acquisition frequency [1].

Secondly, some works address the robot system level. They mainly focus on an identification of the robot energy consumption and energy-aware motion for mission involving a unique control task. In [3], energy consumption predictive models, based on theoretical and experimental analysis of sensors and actuators, are proposed for Khepera III robot. [4] proposes an experimental analysis of power consumption of a Pioneer 3DX (movement energy, sensors and batteries consumption). The authors identify consumption models for DC motor in order to compare the efficiency of different motion plans to cover opened area. Energy prediction models integrating rolling resistance for unmanned ground vehicles is addressed by Sadrpour et al. in [5] to define optimal velocity profile for coverage planning. Optimal velocity for energy efficient navigation has been also addressed in [6] for car-like robots. In [7], minimum-energy trajectory for wheeled mobile robots has been studied and experimented on a Pioneer 3DX.

Few works address the energy question at the mission level involving several types of tasks. In [8], Zhang et al. develop a joint power management scheme coupling the processor frequency (image analysis) and the robot speed (traveling) to improve the whole system power performance. Authors describe in [9] the guidelines for a robot power management system adapting to tasks and environments. Unfortunately few details about its implementation are available. Power-aware tasks scheduling is addressed in [10] for mission-critical embedded systems. However these existing works related to robot energy consumption identification have some limitations:

- Multi-battery (robot and embedded computer) are not considered.

- Impact of different control schemes on energy accuracy is not studied.

- Localization error and environment impact on robot behavior are neglected.

\section{Context}

This work focuses on consumption models, but is part of a more general study on the question of autonomy. Autonomy means also a 'self-awareness' about the performance that the system currently exhibits, or is expected to, depending on mission requirements and environment context. We define different performance axis that has to be monitored in order to guarantee a 'nominal' mission execution from Stability, Safety, Localization, Energy and Duration viewpoints. The originality of our approach consists in the concurrent consideration of global feasibility criteria along these axes based in performance estimation of the different control schemes. In particular, an accurate estimation of the robotic system energy consumption is crucial for energy axis monitoring.

This paper presents in a first part the hardware and software architectures of the robot platform based on a Pioneer 3DX robot. Then the energy consumption of robot and laptop batteries according to the connected elements is experimentally established and modeled. Finally, before concluding, these consumption models are experimentally validated and discussed on a patrolling mission. 


\section{Hardware and Software Architectures}

\subsection{Hardware Architecture}

Based on a classic Pioneer 3DX, the robot platform (Fig. 1 (a)) has been equipped with sensors and electronic devices in order to implement different motion, localization algorithms and image analysis capabilities. So, a rigorous approach of energy management is needed to manage all the control schemes configuration (hard, soft) which can be used.

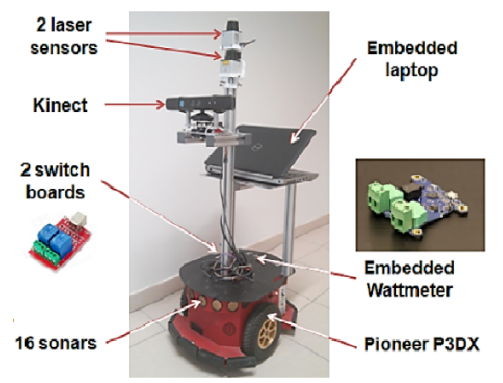

(a)

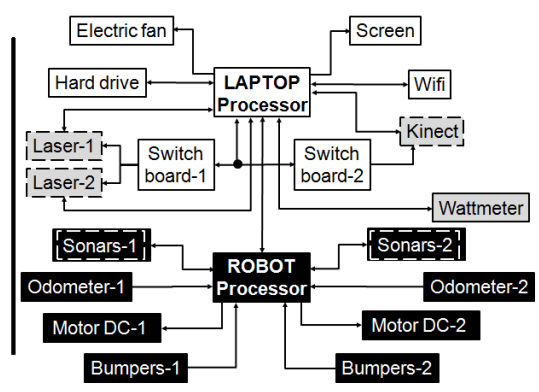

(b)

Fig. 1. (a) Robot platform; (b) Robot hardware architecture.

The Pioneer 3DX is a unicycle type, whose motion can be controlled using two independent DC motors (experimental maximum velocity is $0.8 \mathrm{~m} / \mathrm{s}$ ). It also integrates two arrays (front and rear) of 8 sonars (US) and 5 bumpers. Two URG04 LX Hokuyo lasers (LAS) allow for full horizontal scanning of the environment. Their data are used for obstacle avoidance and robot localization algorithms. A Kinect $^{(}{ }^{(K I N)}$ is used to perform image analysis and robot localization task, using QR-codes. Two added switch boards allow connecting or disconnecting independently the power supply of the two lasers and the Kinect according to the chosen control schemes. The robot embeds also a battery generating up to 259 Wh of energy. An embedded watt-meter has been integrated to realize an online measurement of the energy supplied by the robot battery. The Pioneer 3DX communicates with the embedded laptop using a client-server connection and a Hitachi H8S-based micro-controller.

The embedded laptop includes an Intel-Core i5-2430M CPU 2.40 GHz. Its lithium/ion battery can generate up to $87 \mathrm{Wh}$. This battery supplies the laptop processor, screen, wifi board, hard drive and electric fan. Moreover it also supports the USB communications with the lasers, the Kinect, the switching boards, the watt-meter and the robot micro-controller. The operating system used is Linux-RTAI in order to address real-time issues. The laptop battery consumption is estimated using the linux uevent system file. It allows an online monitoring of the battery current, voltage, and proposes an estimation of the remaining energy of the laptop battery. 
Fig. 1 (b) presents the hardware architecture of the platform. The elements supplied by the robot batteries are drawn with black boxes. The ones supplied by the laptop battery are drawn with white boxes. Switchable components are drawn with dotted lines. Furthermore it must be noticed that these components and the watt-meter have USB connections with the laptop for data exchange but they are supplied from the robot battery (grey boxes).

\subsection{Control Architecture}

The control of the robot behavior, implemented on the laptop, is based on a modular component-oriented hybrid real time control architecture.

This architecture can be decomposed into two main parts. On the one hand, the synchronous executive level is composed of a set of interconnected modules, exchanging data and implementing the robotic basic functionalities using software resources as path-following, obstacle avoidance, corridor centering, for motion control, and laser/US based SLAM and QR-code camera identification or pure odometer for localization. Each possible combination results in a control scheme, recruiting a specific hardware an software set. In case of redundant hardware/software, a set of different alternatives must be considered. It also contains a scheduler, managing modules execution according to real-time constraints and mission objectives. On the other hand, the asynchronous decision level selects the relevant control scheme according to the current mission context using a hierarchy of supervisors. Since the objective of this study is to establish energy consumption models for all possible control schemes, we focus our study on 4 specific control schemes, and their alternatives, inducing the use of different hardware components (Table 1).

Table 1. Control schemes and hardware components

\begin{tabular}{|c|c|c|c|c|c|}
\cline { 3 - 6 } \multicolumn{2}{c|}{} & \multicolumn{3}{c|}{ Sensors } & Actuator \\
\hline \multicolumn{2}{c|}{ Control scheme } & $U S$ & $L A S$ & KIN & DC motors \\
\hline SFM & Simple Forward Motion & - & - & - & $\bullet$ \\
\hline PFM & Path Following Motion & $\circ$ & $\circ$ & $\circ$ & $\bullet$ \\
\hline QRCN & QR Code navigation & $\circ$ & $\circ$ & $\bullet$ & $\bullet$ \\
\hline
\end{tabular}

-: unused / ०: Optional / •: Mandatory

Simple Forward Motion ( $S F M)$ used to measure and identify the energy needed for a straight motion without using sensors or control loop regulation. The two wheels rotation speeds are strictly the same.

Path Following Motion ( $P F M$ ) implements the path-following algorithm SMZ [11] and exteroceptive sensors are recruited. It is used to estimate the control regulation loop impact on energy consumption with respect to the traveling distance and curvature and speed variations. 
QR Code Navigation $(Q R C N)$ allows a global localization of the robot, using the on board camera. Note that the spatial distribution of QR-Codes in the environment influences the positioning error drift and, as a consequence, the path actually performed and the energy consumption.

\section{Energy Consumption Models}

As we presented previously, the studied robot has two power sources: robot battery $\left(P_{R}\right)$ supplies the low-level controller, sensors, motion actuators and watt-meter, while laptop battery $\left(P_{L}\right)$ supplies the control architecture execution, the data exchanged between the processor and the actuators and sensors, and the switch boards. The robot energy management requires building energy consumption models on the robot and laptop batteries.

In the following, the formulation proposed in [3] for a Khepera robot is adapted. Generally, for a given Control Scheme CS, the instantaneous power consumption $P$ of a robot can be decomposed in dynamic and static parts. The dynamic part $P_{D y n}$ denotes the power consumption which can dynamically change over the time. The static part $P_{\text {Stat }}$ denotes the constant steady state power consumption. Depending on the recruited components of a control scheme $C S$, the corresponding instantaneous consumption is:

$$
P(C S)=\sum_{i=1}^{n_{1}} \alpha_{i} \cdot P_{\text {Dyn } i}+\sum_{j=1}^{n_{2}} \beta_{j} \cdot P_{\text {Stat } j}
$$

Where $n_{1}$ is the number of dynamics components, $n_{2}$ the number of static components, $\alpha_{i}$ and $\beta_{j}$ are equal to 1 if the considered component is involved into the current control scheme $C S, 0$ otherwise. For a given control scheme of Table 1 and for a given control scheme, (1) can be rephrased distinguishing optional and mandatory components. The energy consumption of a control scheme is classically obtained by multiplying the instantaneous power consumption by the duration $\Delta T$ of the active control scheme $C S(2)$.

$$
E(C S)=P(C S) \cdot \Delta T(C S) .
$$

Robot and laptop battery consumption models are detailed in the sequel.

\section{Power Model: Consumption on Robot Battery}

We present now a detailed analysis of the robot battery consumption linked to the dynamic and static components. From these results, the power model parameters of the studied control schemes are evaluated. The experimental results are based on the embedded watt-meter data analysis. 


\subsection{Instantaneous Power Consumption of Actuators}

DC motor motion power model for Pioneer 3DX robot has been largely studied in [4], [5], [6] and [7]. Analytically, it depends on many parameters like linear $(v)$ and angular $(r)$ velocities, linear $(a)$ and angular accelerations, robot weight, slope and the type of surface. Model parameters remain difficult to evaluate experimentally and the null acceleration hypothesis is generally observed in the literature [5]. This hypothesis corresponds with the work context where mission is composed by a sequence of straight lines traveled with constant velocities. Another approach developed in [6] and adopted in [3] demonstrates that the DC motor motion power model can be defined by the following polynomial approximation:

$$
P_{R_{-} \text {Motion }}(a, v)=C_{1} \cdot a^{2}+C_{2} \cdot v^{2}+C_{3} \cdot v+C_{4}+C_{5} \cdot a+C_{6} \cdot a \cdot v .
$$

The null acceleration assumption reduces (3) to (4).

$$
P_{R_{-} \text {Motion }}(v)=C_{2} \cdot v^{2}+C_{3} \cdot v+C_{4} .
$$

From experimental measures (Fig. 2 (a)), the polynomial approximation of parameters in (4) yields to (5). This experimental data is the average of power measurements (1 second of period sampling) of $20 \mathrm{~m}$ straight line traveling on a flat horizontal floor using $S F M$ control scheme.

$$
P_{R_{-} \text {Motion }}(v)=6.25 \cdot v^{2}+9.79 \cdot v+3.66 .
$$

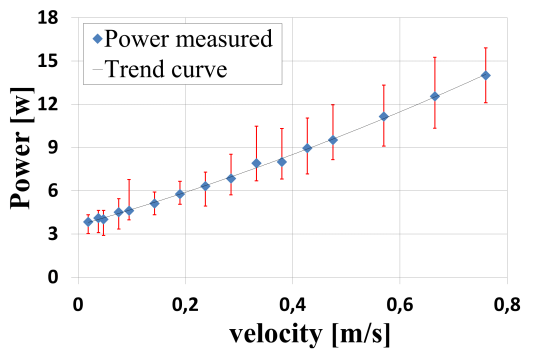

(a)

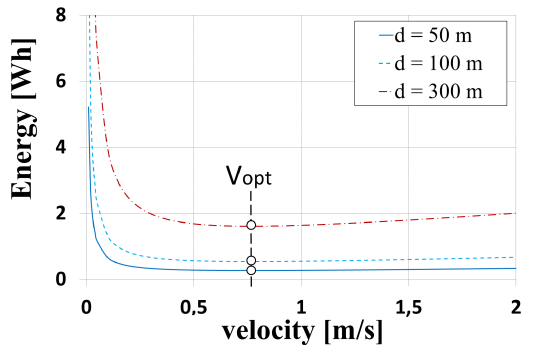

(b)

Fig. 2. (a) DC motor motion power, (b) Theoretical energy for different distances.

Some remarks can be derived from this formulation: when the robot does not move $(v=0 \mathrm{~m} / \mathrm{s})$ the power is equal to $C_{4}(3.66 \mathrm{~W})$. This power $P R_{\text {Cont. } 1}$ corresponds to the static consumption required by the different electronic boards and the micro-controller within the robot. From (2) and (5) and considering that $v=d / \Delta T$ where $d$ represents the traveled distance at a constant velocity $v$ during $\Delta T$, the energy motion model is:

$$
E_{R_{-} \text {Motion }}(d, v)=6.25 \cdot d \cdot v+9.79 \cdot d+3.66 \cdot \frac{d}{v} .
$$


Equation (6) can be very useful to estimate the motion energy needed to travel over a distance $d$ at velocity $v$. Fig. 2 (b) represents the theoretical motion energies required to travel a given distance according to the velocity.

\subsection{Instantaneous Power Consumption of US Sensors}

The power consumption of the sonars is experimentally defined for different acquisition frequencies $f_{U S}$, of the 16 sonars recruitment. Based on these data (Fig. 3), a polynomial curve trend is expressed in (7) where the presented data are the averaged consumption over 60 tests of one minute acquisition.

$$
P_{R_{-} U S}\left(f_{U S}\right)=4 \cdot 10^{-5} \cdot f_{U S}^{2}+5.1 \cdot 10^{-4} \cdot f_{U S} .
$$

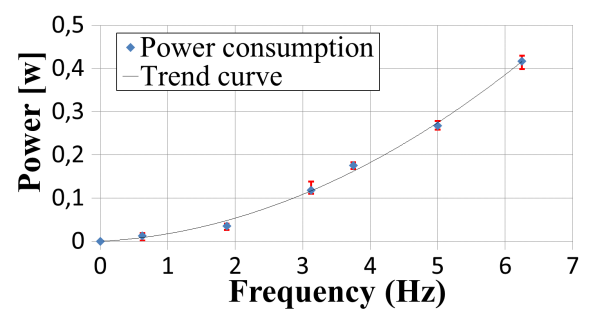

Fig. 3. Sonars consumption.

\subsection{Instantaneous Power Consumption of Static Components}

Power consumption of the static components has been also measured. The most consuming sensor is the Kinect with $P_{R_{-} \text {Kinect }}=2.82 \mathrm{~W}$. Each of the 2 lasers sensors consumes $P_{R_{-} \text {Laser }}=2.34 \mathrm{~W}$. So, using both lasers requires $4.68 \mathrm{~W}$. Finally, the robot controller power consumption is $P_{R_{-} \text {Cont.2 }}=2.67 \mathrm{~W}$.

\subsection{Control Scheme Power Consumption Model for the Robot Battery}

Depending on the components involved in a control scheme, the power consumption extracted from the robot battery can be formulated as:

$$
P_{R}(C S)=P_{R_{-} M o t i o n}(v)+\alpha_{1} \cdot P_{R_{-} U S}\left(f_{U S}\right)+\beta_{1} \cdot P_{R_{-} \text {Kinect }}+k_{1} \cdot \beta_{2} \cdot P_{R_{-} \text {Laser }} .
$$

Where $k_{1} \in\{0,1,2\}$ denotes the number of active lasers. $\alpha_{1}, \beta_{1}$ and $\beta_{2}$ are boolean coefficients that indicate if sensors are used or not. It must be noticed that derivation of (6) and the consideration of (8) yields the following optimal velocity (9). $V_{\text {opt }}=0.76 \mathrm{~m} / \mathrm{s}$ if no sensors used (Fig. 2 (b)). If sensors are used as expressed in (9), the theoretical optimal velocity minimizing the energy consumption cannot be practically reached.

$$
V_{o p t}=\sqrt{\frac{3.66+\alpha_{1} \cdot P_{R_{-} U S}(f)+\beta_{1} \cdot P_{R_{-} \text {Kinect }}+k_{1} \cdot \beta_{2} \cdot P_{R_{-} \text {Laser }}}{6.25}} .
$$




\section{Power Model: Consumption on Laptop Battery}

The laptop battery consumption is generally ignored in the literature. However, in true autonomy context, it is essential. It supports the control architecture and data communication with the robot controller and sensors.

\subsection{Instantaneous Power Consumption of External Devices}

Even if the sensors are externally supplied by the robot battery, the USB communication with the laptop has also its own consumption on the laptop battery (Table 2). Moreover the switching board is also supplied by the laptop battery. That explains why three states are mentioned in the Table 2.

Table 2. Power consumption P of external devices E D

\begin{tabular}{|c|c|c|c|c|c|c|c|}
\hline E D & $\mathbf{P}(\mathbf{W})$ & E D & $\mathbf{P}(\mathrm{W})$ & E D & \multicolumn{3}{|c|}{$\mathbf{P}(\mathrm{W})$} \\
\hline Laser & 0.447 & Kinect & 1.180 & Switch board & 0 & 1 & 2 \\
\hline Controller & 0.075 & Watt-meter & 0.490 & SWILCAI Doda & 0.087 & 0.405 & 0.720 \\
\hline
\end{tabular}

\subsection{Instantaneous Power Consumption of Internal Devices}

The Laptop integrates many internal devices consuming energy. This internal consumption depends on many factors like the executed program (processor activity), hard drive access, electric fan, wifi board consumption, screen display and of course laptop processor and frequency.

The screen power consumption can be easily measured $P_{L_{-} \text {Screen }}=2.69 \mathrm{~W}$. Unfortunately, it is difficult to distinguish the impact of all other factors. However, as supposed in [1] and [9] all these factors can be integrated in unique power consumption $P_{L_{-} \text {Processor }}$ which remains constant for a given control scheme and a given constant control frequency $f_{c}$, as a first approximation. The average value of 60 measurements for different control schemes is: $10.66 \mathrm{~W}(S F M), 13.23 \mathrm{~W}$ $(P F M)$ and $15.45 \mathrm{~W}(Q R C N)$.

\subsection{Control Scheme Power Consumption Model}

The laptop processor frequency is considered as constant, then no dynamic component is involved. The laptop power consumption can also be considered constant for a given control scheme $C S$ and a set of active external components (sensors, switch board). The laptop power consumption can be defined as:

$$
\begin{array}{r}
P_{L}(C S)=P_{L_{-} \text {Processor }}(C S)+\beta_{1} \cdot P_{L_{-} \text {Kinect }}+k_{1} \cdot \beta_{2} \cdot P_{L_{-} \text {Laser }} \\
+\beta_{3} \cdot P_{L_{-} \text {Switch }}\left(k_{2}\right)+\beta_{4} \cdot P_{L_{-} \text {Switch }}\left(k_{3}\right)+\beta_{5} \cdot P_{L_{-} \text {Screen }} .
\end{array}
$$

where, $\beta_{3}, \beta_{4}$ and $\beta_{5}$ are boolean coefficient denoting if the device is used or not. $k_{2}$ and $k_{3}$ indicate whether 0,1 or 2 relays are activated by a switch board. 


\section{$7 \quad$ Experimental Results}

In this section, the proposed energy models applied on the Pioneer 3DX robot and its embedded laptop are validated, traveling along a distance of $50 \mathrm{~m}$ in a corridor from point A to B (Fig. 4). Then, a patrolling mission is considered with more complex path, stops, and different switch between control schemes.

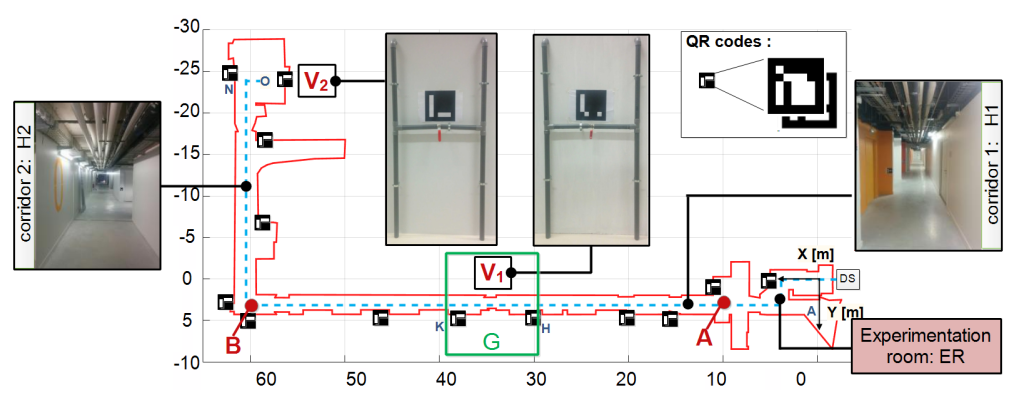

Fig. 4. Experimental context and mission description.

\subsection{Energy Consumption Using Path Following Motion}

A simple forward motion SFM has been used previously to define the power model of the robot motion. Now we will experimentally validate the resulting energy model using different control schemes with path following motion (control loop regulation) with different sensor configurations (supplied but not used in the control loop) and for two distances $(20$ and $50 \mathrm{~m}$ ). Fig. 5 (b) shows the energy measured for robot velocities from 0.1 to $0.8 \mathrm{~m} / \mathrm{s}$.
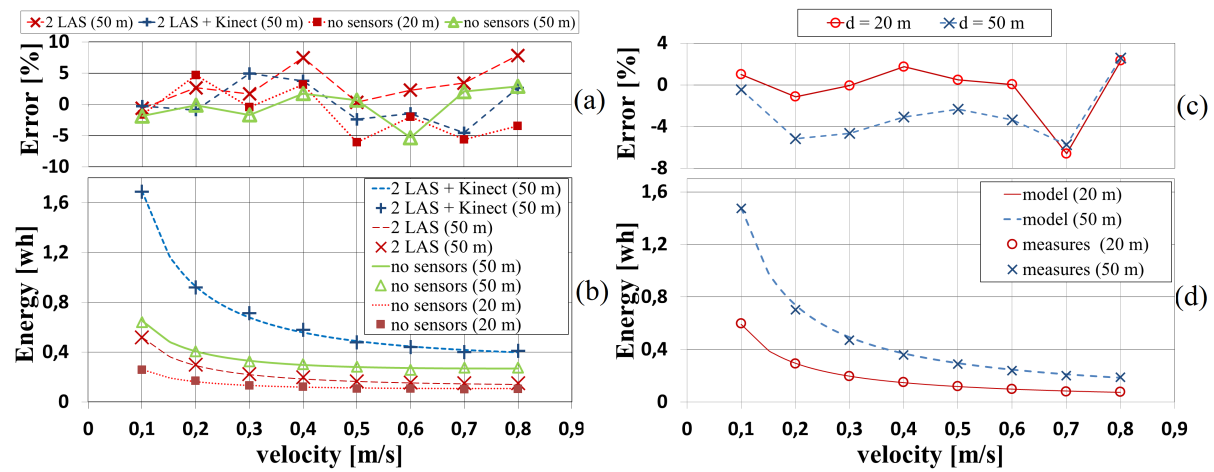

Fig. 5. Path Following Motion: (a) prediction error, (b) experimental and theoretical results for robot energy. (c) Prediction errors, (d) experimental and theoretical results for laptop energy. 
Curves present the theoretical prediction model. The prediction error is computed, and displayed at Fig. 5 (a), where it varies between $-7 \%$ and $+8 \%$. It is interesting to notice that the model accuracy of [3] for a Khepera is very similar to the one we have obtained, despite the large weight difference.

Fig. 5 (c) and (d) present the laptop energy consumed and the corresponding error percentages. Energy measurements fit with model prediction with an error between $-7 \%$ and $3 \%$.

Since the prediction models accuracy can be considered as acceptable on a simple straight line trajectory, it becomes interesting to tackle a mission bringing ground reality like localization errors and acceleration impacts.

\subsection{Patrolling Mission}

A more complex mission is considered in this section. Starting from the docking station (DS) situated in the experimental room ER (Fig. 4), the robot must reach two valves $\mathrm{V} 1$ and $\mathrm{V} 2$ situated in $\mathrm{H} 1$ and $\mathrm{H} 2$ and inspects them (open/closed) by image analysis. Then, the robot must go back to the docking station (starting point). To test the correctness of energy estimation, different velocities are imposed during the mission depending on the robot location: mainly $0.46 \mathrm{~m} / \mathrm{s}, 0.56$ $\mathrm{m} / \mathrm{s}$ and $0.75 \mathrm{~m} / \mathrm{s}$. Fig. 6 presents the experimental measures of the robot velocity during the proposed mission and the mean velocity reached in each area is indicated in blue over the red lines (theoretical velocity). Two stops are noticed for image analysis detecting the valves status.

The total mission duration is higher than 7 minutes (434 seconds) over $190 \mathrm{~m}$ traveled distance. A path following control scheme QRCN (CS 1) using kinect (localization) has been used with sonars and two lasers (obstacle avoidance). On the way back, switching off one laser is planned (CS. 2). Moreover into the valves areas, a pure rotation is triggered OPR (CS. 3) and image analysis IA (CS. 4) control scheme is used to steer the robot in front of the valve and to analyze its status (open / close). The use of these schemes is shown in Fig. 6.

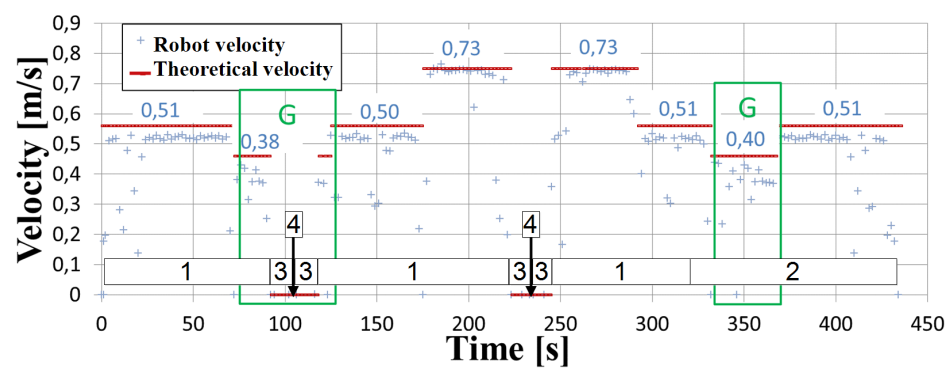

Fig. 6. Mission experimental velocity variations.

Fig. 7 (a) shows that experimental robot energy consumption is a little bit higher than the expected one. However the curves are very closed. Moreover 
Fig. 7 (b) demonstrates that the predicted energy estimation error after an initial peak decreases to a final value of $10 \%$ (12.1\% for laptop battery Fig. 7 (d)). The initial over-consumption is due to the system trajectory to reach the first corridor from the ER. The real trajectory differs from the expected one (odometer drift and robot oscillations) and the real consumption is higher than the predicted one. The same increase of error is noticed at the end of the mission (from $350 \mathrm{~s}$ ) where robot returns to the ER. The same peaks are noticed for the laptop prediction error. Moreover, the over-estimation of the energy consumption (at the beginning of the mission) becomes less important with regards to the global energy consumption according to the mission progress (error decrease). The estimation error is explained also by the difference between the theoretical and the experimental velocities and the robot accelerations after the stops near the valves.
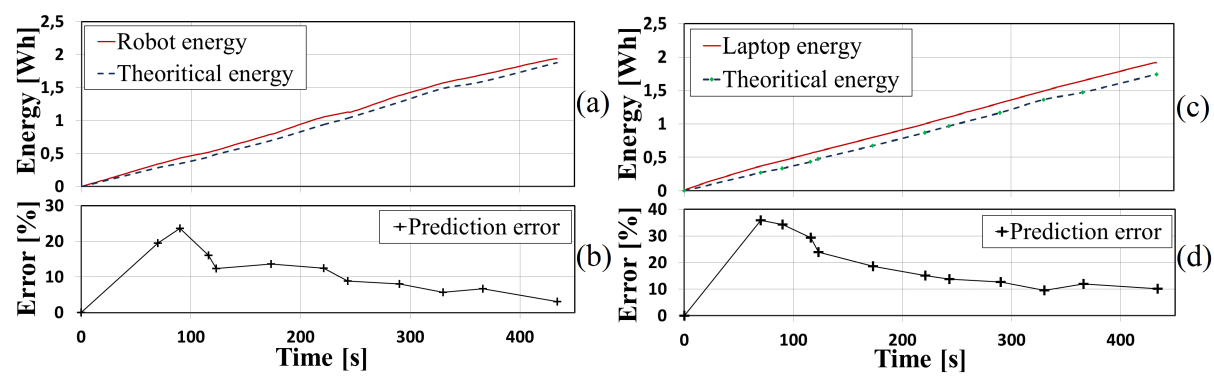

Fig. 7. Robot energy consumption: (a) Experimental and theoretical results, (b) prediction error. Laptop energy consumption: (c) Experimental and theoretical results, (d) prediction error.

These analysis demonstrate that the proposed power and energy consumption models allow for a good estimation of the real consumption for quite complex and long missions. The over-estimation observed is due to the difference between the expected and real robot trajectories. This difference is due mainly to the system transient response and the robot odometer drift for quite long distance before being corrected by QR code localization (robot oscillation and velocity variations). When robot heading changes are not frequent the consumption estimation becomes close to the reality.

\section{Conclusion}

This paper tackled the question of energy prediction of the Pioneer 3DX robot in connection with energy management for autonomous mobile robotics. Energy aware based on accurate energy consumption estimation becomes crucial. Then, both robot and embedded laptop where considered. Based on experimental analysis, generic energy prediction models were proposed for a given control scheme. 
Optimal velocity from robot energetic viewpoint is defined. Experimental results show that the measurements fit to the proposed models for path following motion with different control schemes using different sensors. Later, a more complex mission is realized and the impact of the localization accuracy and the path shape are noticed. Thus, prediction accuracy decreases but remains still acceptable.

Future works, look for identifying the gap between prediction and experimental results tacking in account localization accuracy and followed path. That will improve the prediction models accuracy. Based on this prediction, a methodology allowing energy management for autonomous robotic mission has been developed. This methodology consists on dynamic hardware and software resources allocation along the mission with regard to performance constraints and particularly the energy one.

\section{References}

1. Mei, Y., Lu, Y.H., Hu, Y.C., George Lee, C.S.: A Case Study of Mobile Robot Energy Consumption and Conservation Techniques. In: Proc. of the 12th International Conference on Advanced Robotics (ICAR'05), pp. 492-497 (2005)

2. Pillai, P., Shin, G.C.: Real Time Dynamic Voltage Scaling for Low-Power Embedded Operating Systems. In: Proc. ACM 18th ACM Symp.Operating Systems Principles, Banff, Alberta, Canada (2001)

3. Parasuraman, R., Kershaw, K., Pagala, P., Ferre, M.: Model Based On-line Energy Prediction System for Semi-Autonomous Mobile Robots. In: Proc. of the 5th International Conference on Intelligent Systems Modeling a Simulation, pp. 27-29 (2014)

4. Mei, Y., Lu, Y.H., Hu, Y.C., George Lee, C.S.: Energy-Efficient Motion Planning for Mobile Robots. In: Proc. of the International Conference on Robotics \& Automation, pp. 4344-4349 (2004)

5. Sadrpour, A., Jin, J., Ulsoy, A.G.: Mission Energy Prediction for Unmaned Ground Vehicles Using Real-time Measurements and Prior Knowledge. Journal of Field Robotics, 30(3), pp. 399-414 (2013)

6. Tokekar, P., Karnad, N., Isler, V.: Energy-optimal Velocity Profiles for Car-Like Robots. In: Proc. IEEE Int. Conf. on Robotics and Automation - ICRA 2011. pp. 1457-1462 (2011)

7. Kim, C.H., Kim, B.K.: Minimum-Energy Motion Planning for differential-Driven Wheeled Mobile Robots. In Motion Planning INTECH Open Access Publisher, pp. 193-226 (2008)

8. Zhang, W., Lu, Y.H., Hu, J.: Optimal Solutions to a Class of Power Management Problems in Mobile Robots. In Automatica, 45(4), pp. 989-996 (2009)

9. Ogawa, K., Kim, H., Mizukawa, M., Ando, Y.: Development of the robot power management system adapting to tasks and environments-The design guideline of the power control system applied to the distributed control robot. In: Proc. of the 2006 SICE-ICASE International Joint Conference, pp. 2042-2046 (2006)

10. Liu, J., Chou, P.H., Bagherzadeh, N., Kurdahi, F.: Power-aware scheduling under timing constraints for mission-critical embedded systems. In: Proc. of the 38th Design Automation Conference, pp. 840-845 (2001)

11. Lapierre, L., Zapata, R.: A guaranteed obstacle avoidance guidance system: The safe maneuvering zone. In Autonomous Robots, Springer Verlag (Germany), pp.177$187(2012)$ 